\title{
Pratiques
}

Linguistique, littérature, didactique

183-184| 2019

oralité, littératie

\section{Continuum anthropologique}

Jean-Marie Privat

\section{(2) OpenEdition}

Journals

Édition électronique

URL : http://journals.openedition.org/pratiques/6742

DOI : $10.4000 /$ pratiques. 6742

ISSN : 2425-2042

\section{Éditeur}

Centre de recherche sur les médiations (CREM)

Référence électronique

Jean-Marie Privat, "Continuum anthropologique », Pratiques [En ligne], 183-184 | 2019, mis en ligne le 30 décembre 2019, consulté le 11 octobre 2020. URL : http://journals.openedition.org/pratiques/6742 ; DOI : https://doi.org/10.4000/pratiques. 6742

Ce document a été généré automatiquement le 11 octobre 2020

(c) Tous droits réservés 


\title{
Continuum anthropologique
}

\author{
Jean-Marie Privat
}

1 La problématique du continuum anthropologique a été largement esquissée dans un précédent numéro de Pratiques (Privat \& Kara, 2006) consacré à la littératie. Il y est question de prendre en compte le fait qu'il y a des degrés dans la littératie et par conséquent dans l'oralité : "Il est manifeste par exemple que le dictionnaire, le catalogue, le billet d'avion électronique, l'index, le roman, la table de multiplication, la carte, la réécriture, la relecture, la prise de notes, etc. présentent un très fort taux de littératie. Et inversement, "observer une minute de silence", "maudire" ou "chanter une berceuse" sont des rites fondamentalement oraux qui n'ont pas d'équivalents pragmatiques à l'écrit ». Ces polarités présentent l'intérêt de déplacer la question du code (phonique/acoustique versus graphique/visuel) et d'introduire à une conception praxéologique des modalités de la communication. La plupart des usages langagiers présentent en effet des taux ou gradients de littératie plus ou moins convertibles à l'oral, ou se conjuguent avec des moments d'oralité. Ainsi une lettre d'amour ne saurait être confondue avec une déclaration d'amour en face to face, même si dans certains cas l'une peut se substituer ou préluder à l'autre. Et si dans les cérémonies républicaines de mariage la signature - de sa propre main - scelle officiellement le contrat, la présence physique des témoins est requise tout comme la profération rituelle d'un sonore et attendu «Oui ». Ces hybridations montrées ou constitutives sont peut-être plus nombreuses et plus constantes qu'une conception monolithique et canonique de la littératie ne le laisserait accroire : «Ce continuum dans les modes de communication est parfois à ce point incorporé que l'orateur peut "parler comme un livre", " ponctuer son discours ", «mettre les points sur les $i$ » et que l'écolier modèle qui a goûté à « la soupe à l'alphabet » et fait ses premiers apprentissages en lecture dans des abécédaires anthropomorphes (ou zoomorphes) se doit de réciter "par cœur" le justement nommé ordre alphabétique ou telle fable de La Fontaine, sans en changer une virgule. ${ }^{1}$

2 Il convient de compléter ces observations en renvoyant aux notices «société orale société écrite » et " polylogie» de notre abécédaire et sans doute d'éclaircir d'autres aspects du continuum anthropologique. 
Un exemple d'oralité formulaire suffira ici. Certes l'univers du tribunal est régi par le Code civil, monument de littératie. Et les avocats, spécialistes de la parole oratoire, sont pétris de culture juridique écrite. Le serment que doit prêter un témoin ou un accusé, cités à comparaître dans un procès présente une autre facette de la situation énonciative. La personne prête serment publiquement. Elle est debout, fait face aux juges souvent en surplomb, en lieu et place réglementaire. La formule explicitement requise est dite ne varietur à haute et intelligible voix, dans la langue officielle. Bref, le protocole de la coprésence des actants, accompagnée d'une distance physique et plus encore symbolique, institue un cadre communicatif. Mais, si le serment du témoin - « Je jure de dire toute la vérité, rien que la vérité »- est bien un «dire " aux effets performatifs - « dire » c'est « être » (témoin) et « faire » (serment) - cet acte de langage ne se suffit pas manifestement à lui-même. Une synchronie énonciative engage une sémiotique du corps et du geste, main droite ostensiblement levée et ouverte. Le continuum anthropologique s'actualise dans la coalescence expressive d'un geste/mot ou d'une phrase-geste. On ne saurait dire que le geste vu accompagne le verbe entendu, pas plus que l'inverse (ordinairement désigné comme "paralinguistique »). Il s'agit d'un emboitement de deux modes sémiotiques, une mise en forme linguistique et une mise en forme somatique. C'est cette intégration de microsystèmes co-signifiants ${ }^{2}$ qui sans hiérarchie - fait de facto et de jure l'événement communicatif. Ce continuum d'oralité renvoie à bien d'autres formes de corps-oralité où l'articulation corporelle somatise de façon subliminale le langage et où le langage articulé surmotive l'adresse du corps. Cette oralité syncrétique est en effet déjà - par exemple - typique de l'imaginaire langagier de l'enfant pour qui le mot « tétine » est en symbiose avec l'objet lui-même ou chez qui le propre prénom est - et demeurera... - comme incorporé à/par la personne privée et publique qui l'incarne. Un continuum anthropologique en quelque façon entre oralité personnelle et oralité solennelle. On pourrait analyser sur le même mode du continuum la pratique de la signature manuscrite qui combine un engagement scriptural fiduciaire in praesentia, une énonciation idiographique et un geste idéographique, une pulsion sémiotico-somatique contrôlée et incorporée (une " griffe " plus visible que lisible, un ensauvagement communicationnel codé), enfin un puissant investissement imaginaire dans le totem d'une déliaison graphique et de ses pouvoirs. Une auto-bio-graphie - souvent - infra-linguistique en somme. Il suffirait enfin de penser à l'immense et incessant archipel des discours intérieurs, "paroles " inarticulées dont le ressassement échappe parfois à la claire volonté du sujet diurne ou nocturne; performances langagières en procès sous forme de boucles dialogiques entre oralité intime et logosphère normée - et où les échanges verbaux avec soi-même préparent une prise de parole ordonnée ou un passage calculé à l'acte d'écriture. Dans l'un ou l'autre cas, la discursivité de la langue opère selon un moyen de production proche d'un proto-oral et des modes de réalisation de la parole vive ou de la textualité écrite ${ }^{3}$. Un continuum subliminal et indécidable en règle générale. 


\section{BIBLIOGRAPHIE}

BENVENISTE, É. (2012). Dernières leçons. Collège de France 1968 et 1969. Paris : Éditions de l'EHESS/ Gallimard/Seuil.

KERBRAT-ORECCHIONI, C. (2016) [2001]. Les actes de langage dans le discours. Théorie et fonctionnement.

Paris : A. Colin.

LAFONT, R. (1994). Il y a quelqu'un. Le langage et le corps. Montpellier : Praxiling.

PRIVAT, J.-M. (2006) « Un habitus littératien ? ». Pratiques 131-132, p. 125-130. En ligne : https://

www.persee.fr/doc/prati_0338-2389_2006_num_131_1_2122.

PRIVAT, J.-M. \& KARA, M. (2006). Pratiques 131-132. En ligne : https://www.persee.fr/issue/

prati_0338-2389_2006_num_131_1.

\section{NOTES}

1. Privat, 2006.

2. Nous rejoignons ici l'anthropologie linguistique de Robert Lafont (1993) quand il vise à retrouver "l'unité de la fonction signifiante » et qu'il analyse comment « le thème linguistique s'unit à un thème somatiquement exprimé. » Cette " actualisation polyorganique» est multi-sensorielle et pluri-sémiotique. On retrouverait une problématique voisine chez Catherine Kerbrat-Orecchioni (2016 [2001], p.151) lorsqu'elle note que les « actes non langagiers » fonctionnent « en symbiose " avec les «actes langagiers» et qu'elle fait observer que si la communication est bien un "tout intégré", rares sont encore les descriptions des interactions qui en tiennent compte.

3. On se souvient qu'à propos de «la conversion en écrit », pour Benveniste (2012, p. 94), « l'acte d'écriture ne procède pas de la parole prononcée, du langage en action, mais du langage intérieur, mémorisé [...]. Le langage intérieur a un caractère global, schématique, non construit, non grammatical. C'est un langage allusif. »

\section{AUTEUR}

\section{JEAN-MARIE PRIVAT}

Université de Lorraine, Crem, F-57000 Metz, France 